
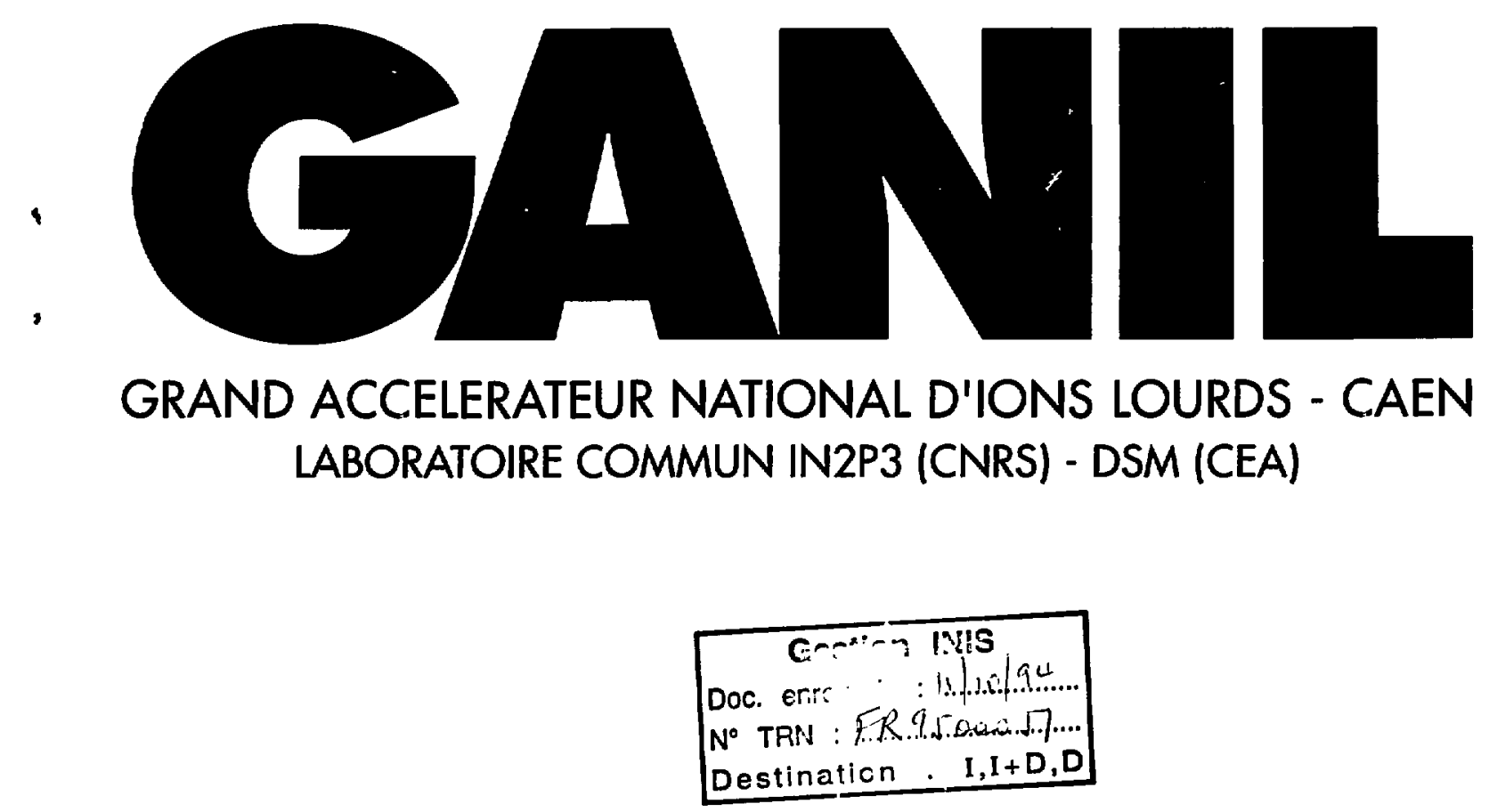

APPLICATION TO THE SPIRAI. PROJECT AT GANIL OF A NEW KIND OF LARGE ACCEPTANCE MASS SEPARATOR.

B.BRU, A.CHIABERT, Ch.RICAUD.

GANIL-B.P.5027 F-14021 CAEN-CEDEX.

$4^{\text {th }}$ International Conference on Charged Particle Optics October 3-6, 1994, Tsukuba, Japan

GANIL S 9402

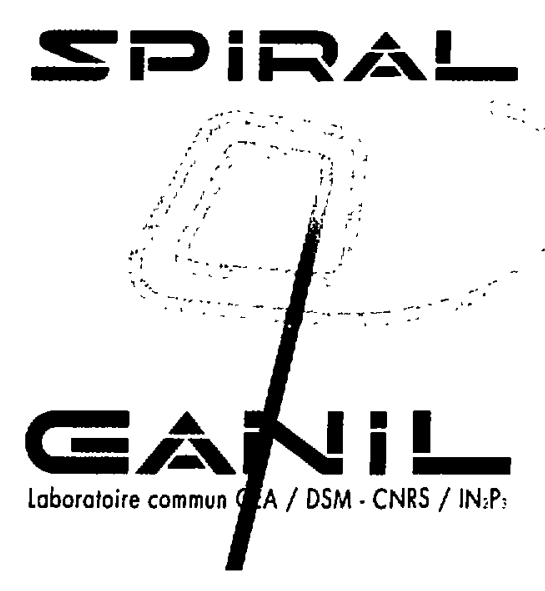




\title{
APPLICATION TO THE SPIRAL PROJECT AT GANIL OF A NEW KIND OF LARGE ACCEPTANCE MASS SEPARATOR.
}

\author{
B.BRU, A.CHABERT, Ch.RICAUD. \\ GANIL-B.P.5027 F-14021 CAEN-CEDEX.
}

\section{1- INTRODUCTION.}

The SPIRAL project under construction at GANIL [1] aims to post-accelerate Radioactive Ion Beams (RIB) using a cyclotron. The radioactive atoms are produced in the interactions of the high energy, high intensity heavy ion beams delivered by GANIL inside a thick target (ISOL method). They are ionized using an ECR ion source and extracted at a maximum voltage of $30 \mathrm{kV}$.

These low energy RIB of large emittance (up to $150 \pi \mathrm{mm} . \mathrm{mrad}$ ) are composed of a large number of ion species, some of them having similar $\varepsilon=Q / M$ ratios, whose relative intensities may range from 1 to even more than $10^{6}$.

Before injection into a post-accelerator it is of prime importance first to identify the required isotope among a variety of other ones and to optimize its production, then to restrict the number of ion species injected into the cyclotron and, if possible, to perform an on-line control of the production. Of course we would like to minimize the beam losses during these operations.

To perform these functions we intend to use a new kind of Large Acceptance Mass Separator (LAMS) proposed by the authors [2].

\section{2 -THE SPIRAL FACILITY.}

\subsection{Generalities.}

The layout of the present SPIRAL project is shown in fig.1 :

- two heavily shielded "target-ion source" systems are located in the basement and receive the primary beam ;

- a low energy beam line transports the secondary RIB from the ion sources to the cyclotron inflector : this line is divided into three parts :

. two identical parts, one per ion source : each part includes an achromatic mass spectrometer followed by a common matching section to the third part. The mass resolution is better than $\cong 200$ for a $80 \pi \mathrm{mm}$.mrad radial emittance. of the cyclotron.

. the third part is devoted to the $6 \mathrm{D}$ matching of the beam on the first orbit

- a compact cyclotron $(K=265)$ gives the radioactive ions an energy ranging from 2 to $25 \mathrm{MeV} /$ nucleon. It is designed to accept beams of $80 \pi \mathrm{mm} . \mathrm{mrad}$ in each 
transverse plane and $\pm 6^{\circ}$ in RF phase. The working diagram is shown in fig.2.

- a medium energy beam line transports the beam extracted from the cyclotron to the high energy spectrometer (Alpha Spectrometer) and then to the experiments.

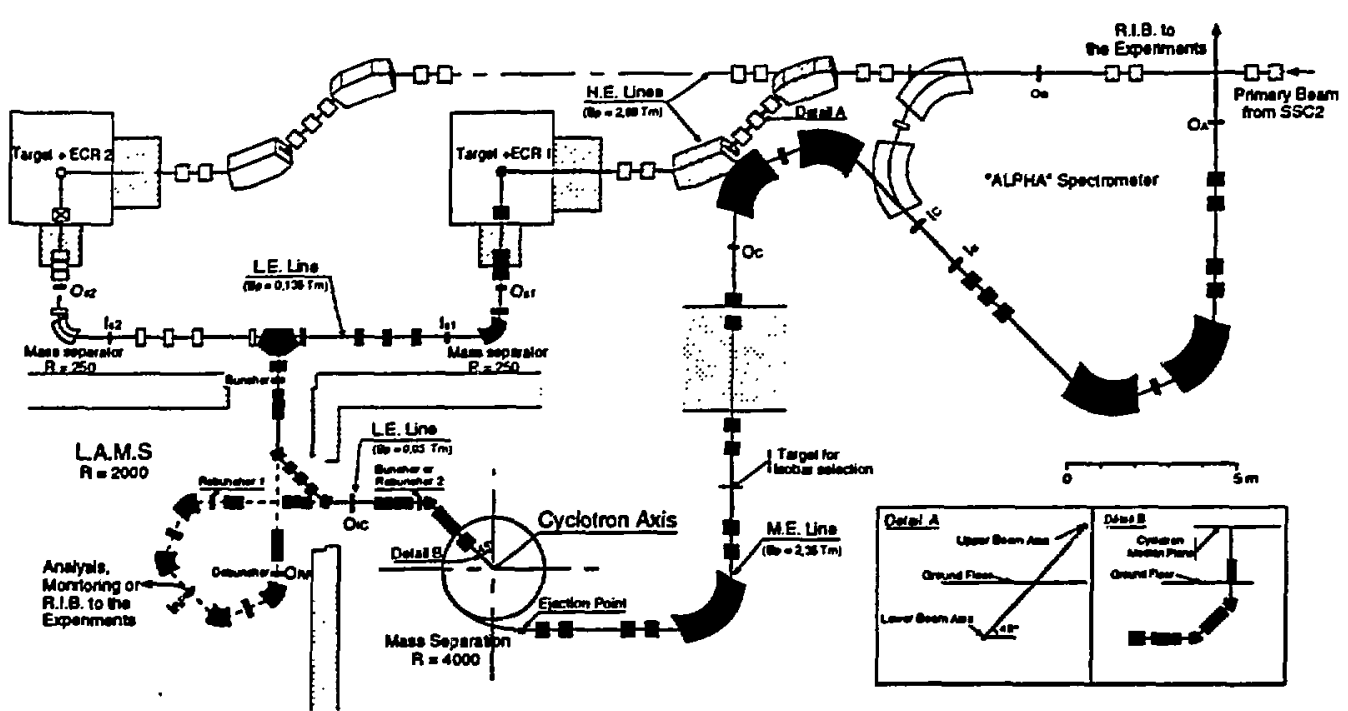

Figure 1 : Layout of the beam lines of SPIRAL.

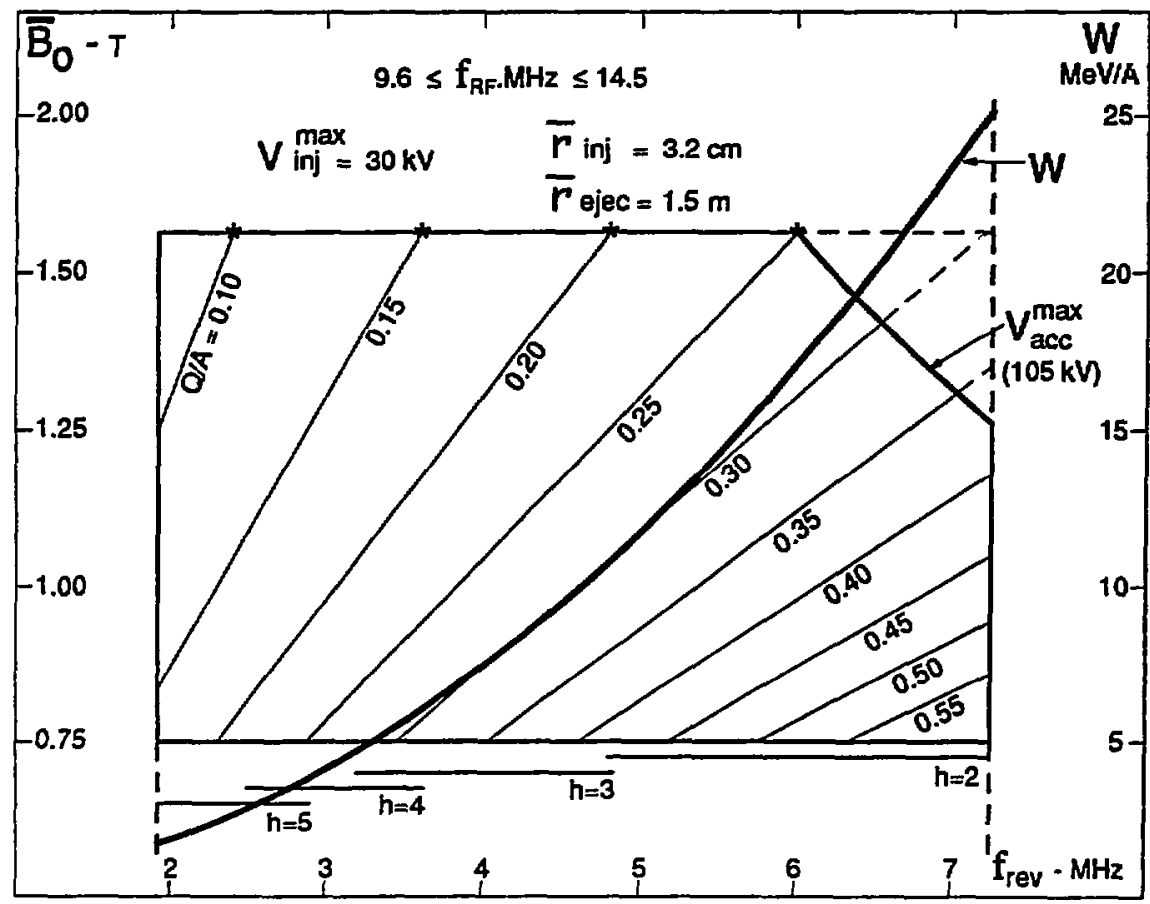

Figure 2 : Working diagram of the cyclotron.

We propose to insert the new mass separator into the low energy beam line, between its first two parts and the third one. In fig.1, this separator appears in dotted lines. 


\subsection{The low energy beam line (see fig.1).}

All the guiding and the transverse focusing elements of this line (dipoles, quadrupoles, sextupoles and solenoids) are magnetic.

\section{a) The first two parts.}

Taking into account the characteristics of the beam extracted from the ECRIS, these parts fulfil two main functions : they ensure a rough mass analysis using anti symmetrical achromatic spectrometers and allow to define the transverse emittance. The betatron matching at the object point of each spectrometer (maximum transverse emittance of $150 \pi \mathrm{mm} . \mathrm{mrad}$ ) requires 4 elements : a solenoid followed by a triplet.

The analysers are double focusing magnets $\left(\theta=90^{\circ}, \rho=0.5 \mathrm{~m}\right)$; each one is followed by a symmetrical triplet. The common dipole of compensation is a twofold magnet $\left(\theta=90^{\circ}, \rho=0.5 \mathrm{~m}\right)$ allowing to transport the beam from both ECRIS with similar optics ; the pole face angle at the exit of this dipole being $0^{\circ}$ the vertical focusing is provided by a quadrupole placed just after. This quadrupole and ... triplet perform the betatron matching at the object point of the third part through an achromatic deviation composed of a symmetrical triplet between two $45^{\circ}$ rectangular dipoles of $p=0.35 \mathrm{~m}$.

A sextupole is placed at the entrance of each $90^{\circ}$ dipole in order to compensate for the second order geometrical aberrations.

\section{b) The third part.}

This part is the axial injection line in the cyclotron : with a structure similar to that of the GANIL Injector [3], it allows to match the beam on the first orbit in the 6D phase space and to optimize the injection yield.

Assuming a Müller-type hyperboloidal inflector (the solution presently selected), this part fulfils the following functions : the betatron matching using a quadruplet, the radial chromatic matching by means of a $45^{\circ}$ dipole and a triplet, the vertical chromatic matching using two $45^{\circ}$ vertical dipoles with a triplet in between. A solenoid located in the cyclotron yoke adjusts the rotation of the beam at the entrance of the inflector. These $45^{\circ}$ dipoles are pure sectors of $\rho=0.35 \mathrm{~m}$.

\section{c) The bunching system.}

We intend to use a gridded single gap buncher giving an almost linear modulation over $\equiv \pm 135^{\circ}[4,5]$. It has to be located before the horizontal $45^{\circ}$ deviation of the injection line in order to give the required correlations at the entrance of the cyclotron.

This beam line has been optimized to first order using the GALOPR code [6] and the resulting monochromatic transverse beam envelopes from the object point of the $90^{\circ}$ analysers to the exit of the cyclotron inflector are given in fig.3. 


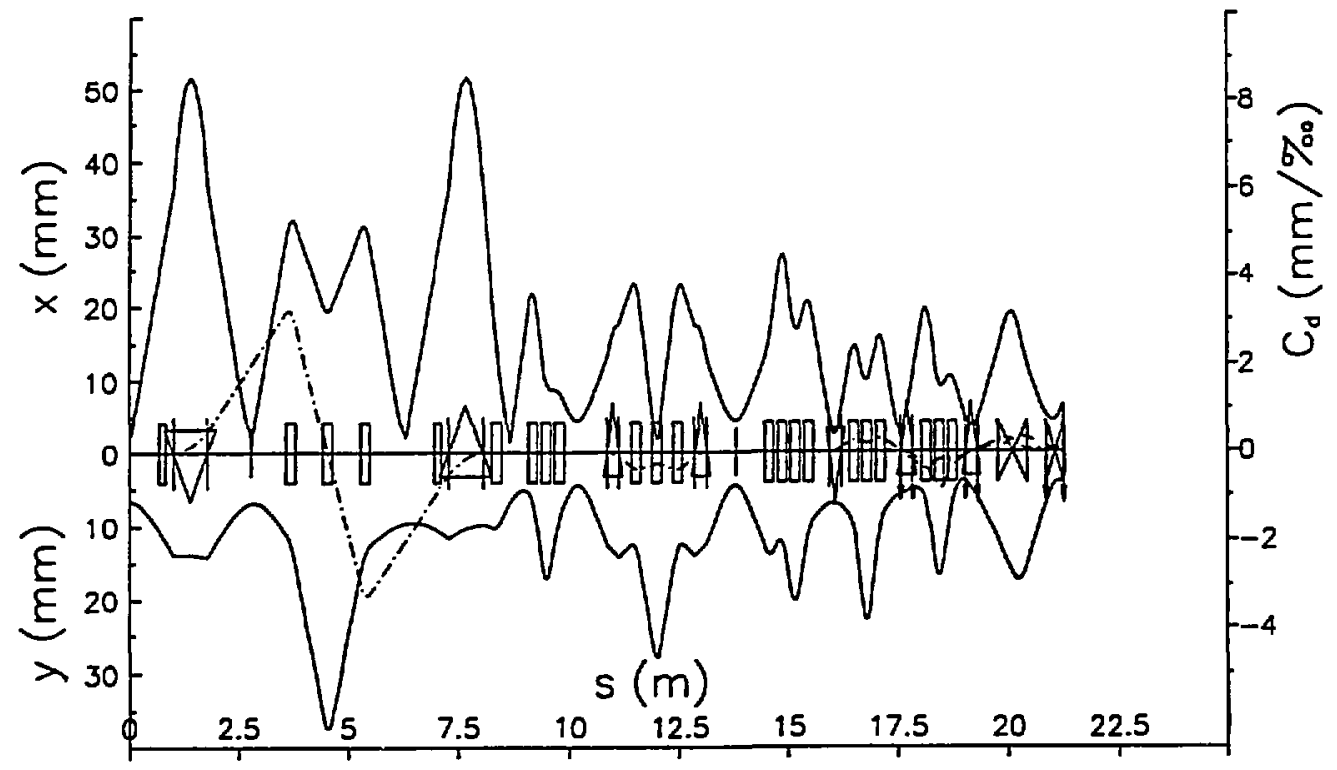

Figure 3 : monochromatic beam envelopes in the low energy beam line as planned in the SPIRAL project ( $80 \pi \mathrm{mm} . \mathrm{mrad}$ in each transverse plane ).

\section{3 -THE INSERTION OF THE LARGE ACCEPTANCE MASS SEPARATOR.}

\subsection{Principle of the mass separator.}

The principle of this mass separator is illustrated in fig.4.

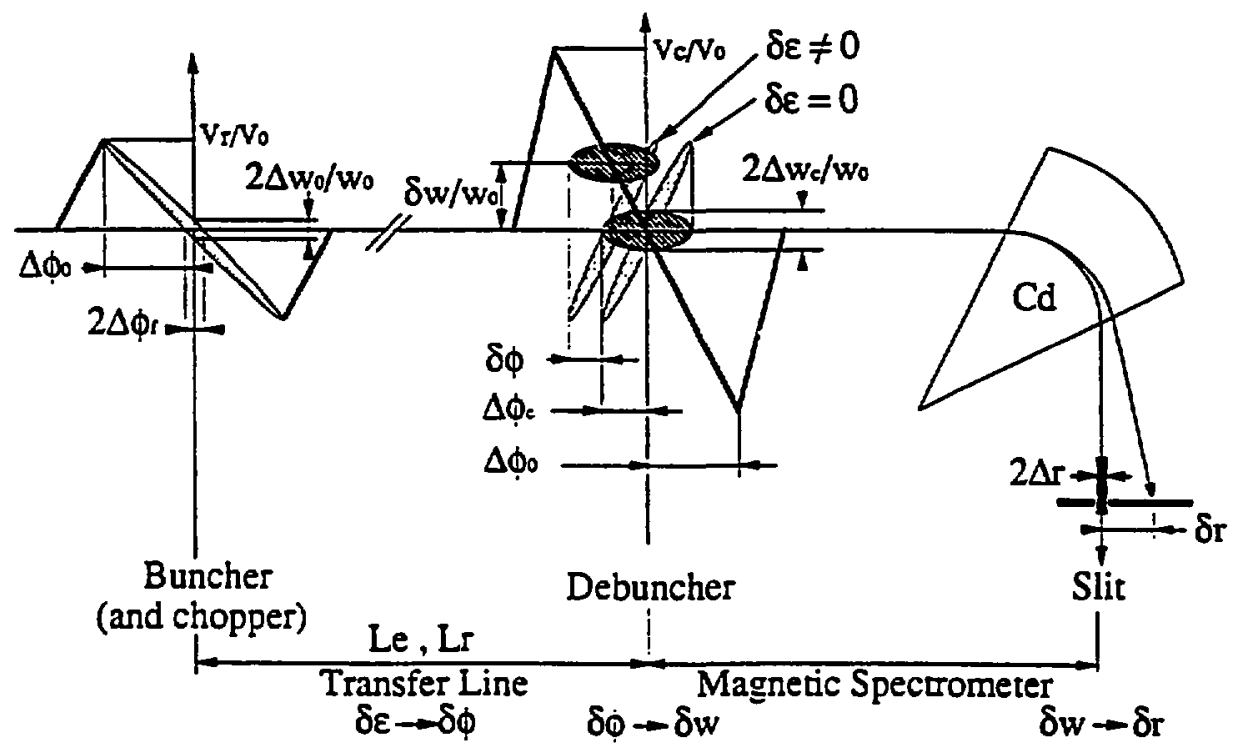

Figure $4:$ The principle of the mass spectrometer. 
Such a mass separator can be divided into three successive sections :

- the first one includes a buncher and a transport line equipped with the suitable transverse focusing elements. In this section the various kinds of ions are bunched in the same phase width around a mean phase depending on their charge-to-mass ratio $\varepsilon$.

- the second section is a debuncher which transforms the mean phase shifts into mean energy shifts while minimizing the energy spread of each component.

- in the third section a magnetic analyser transforms the mean energy shifts into mean radial position shifts while minimizing the radial width of each component. A radial slit at the image point of the system selects the required ions.

We can imagine various kinds of transport line, the two extreme cases being :

- a straight drift : in this case the bunching length $L_{e}$ is equal to the drift length $L_{\tau}$,

- an isochronous transport $\left(\mathrm{L}_{\mathrm{e}}=0\right)$ : in this case the initial beam has to be bunched and matched in 6D at the entrance of the line.

Of course any achromatic transport (the beam itself being achromatic at the entrance) is convenient, a line with $\mathrm{L}_{e}<\mathrm{L}_{\boldsymbol{T}}$ being specially interesting.

We choose the simplest case of a straight drift $: L_{e}=L_{r}=L$.

At the entrance of the system, the achromatic $\mathrm{cw}$ beam contains ions with different $\varepsilon$ values which have been accelerated through the same static potentiel $V_{0}$.

Assuming the buncher and the debuncher voltages (respectively $V_{r}$ and $V_{c}$ ) linear over a $\pm \Delta \phi_{0}$ phase range (theoretical yield $\Delta \phi_{0} / \pi$ ), we find, in the linear approximation, the lowest $\mid \delta \varepsilon / \varepsilon_{0} /$ which can be analysed ; it is given by [2] :

$$
\left|\frac{\delta \varepsilon}{\varepsilon_{0}}\right| \geq \frac{\left\{\left[\frac{4 \mathrm{e}_{0}}{\mathrm{C}_{\mathrm{d}}}\left(\frac{\mathrm{L}}{\beta \lambda} \frac{\mathrm{V}_{\mathrm{r}}}{\mathrm{V}_{0}}-\frac{\Delta \phi_{0}}{\pi}\right)\right]^{2}+\left[2 \frac{\Delta \phi_{0}}{\pi} \frac{\Delta \mathrm{w}_{0}}{\mathrm{w}_{0}}\right]^{2}\right\}^{1 / 2}}{\frac{\mathrm{L}}{\beta \lambda} \frac{\mathrm{V}_{\mathrm{r}}}{\mathrm{V}_{0}}}
$$

where : $\Delta \phi_{0} \times \Delta w_{0} / w_{0}$ is the longitudinal emittance at the entrance of the line,

$\beta \lambda$ is the particle wave length at the common frequency of the buncher and debuncher,

$C_{d}$ and $e_{o}$ are the radial momentum dispersion coefficient and the monochromatic radial half beam width at the image point of the spectrometer respectively.

Fig.5, deduced from this inequality, gives the $1 \delta \varepsilon / \varepsilon_{0} l$ one can separate using this system. As can be seen, the working point of LAMS has obviously to be chosen around $(\mathrm{L} / \beta \lambda)\left(\mathrm{V}_{\mathrm{r}} / \mathrm{V}_{0}\right)=(4 / 3)\left(\Delta \phi_{0} / \pi\right)$. For a voltage linear over $\Delta \phi_{0}=135^{\circ}$, we should obtain $\Delta \phi_{c}=45^{\circ}, \Delta w_{c} / w_{0}=3 \Delta w_{0} / w_{0}$ and a separation slightly over $2 \Delta w_{0} / w_{0}$ depending on the radial emittance through $e_{0} / C_{d}$. 
In a real system this separation will be reduced due in particular to the non linear effects (geometrical and magnetic aberrations)

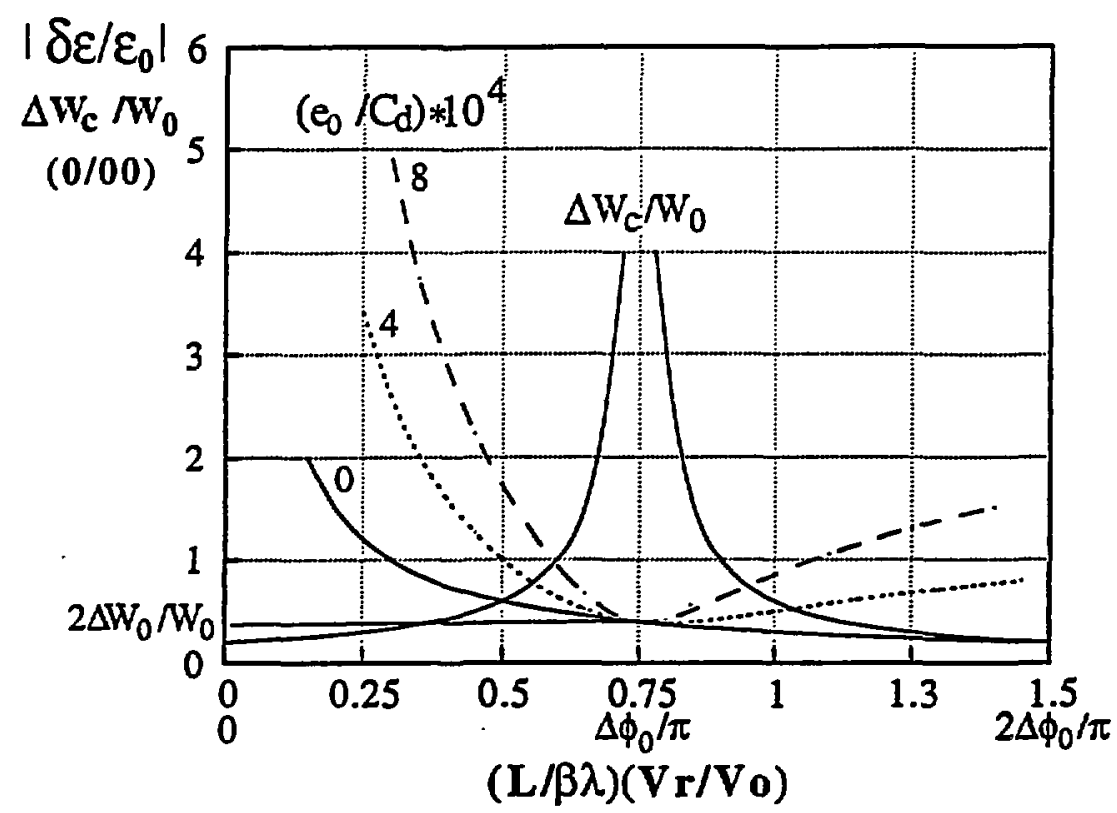

Figure 5 : first order characteristics of a $\mathrm{L}_{\mathrm{e}}=\mathrm{L}_{\mathrm{T}}=\mathrm{L}$ mass separator. $\left|\delta \varepsilon / \varepsilon_{0}\right|$ for $\mathrm{e}_{0} / \mathrm{C}_{\mathrm{d}}=0,4,810^{-4}$ and $\Delta \mathrm{w}_{\mathrm{c}} / \mathrm{w}_{0}$ versus $(\mathrm{L} / \beta \lambda)\left(\mathrm{V}_{\mathrm{r}} / \mathrm{N}_{0}\right)$

$$
\left(\Delta w_{0} / w_{0}=2 \times 10^{-4}, \Delta \phi_{0} / \pi=0.75\right)
$$

In our approximation, the separation is obtained modulo $2 \pi$ on the mean phase shift so that, depending on the $\varepsilon$ range to be analysed, a first rough spectrometer could be required.

\subsection{Description of the proposed mass separator.}

Instead of going through the double $45^{\circ}$ deviation the beam follows the dotted line (fig.1) which is the achromatic LAMS.

The transport line includes two triplets, the first one being the one following the twofold magnet of compensation. This symmetrical focusing structure is tuned to obtain at both ends two radial waists having the same radial dimensions.

The achromatic spectrometer is "alpha shaped", it is composed of 2 pairs of dipoles $\left(\theta=67.5^{\circ}, \rho=0.5 \mathrm{~m}, \eta=18^{\circ}\right)$ giving a total deviation of $270^{\circ}$ and providing a plane of symmetry. The distance between the two dipoles of a same pair has been adjusted to give a radial angular dispersion coefficient equal to 0 between the two pairs of dipoles so that this achromatic spectrometer of $C_{d}=1.94 \mathrm{~mm} / \%$ o does not need any quadrupole.

For topological reasons and to preserve the complete symmetry of the whole system, the object distance is chosen shorter than the image one, the monochromatic radial beam size is the same at the object, at the image and at the waist behind the last 
dipole. In this system, the bunching length $(2.20 \mathrm{~m})$ is much shorter than the geometrical length $(7.07 \mathrm{~m})$ and at its exit the beam remains well bunched in phase. Therefore we can place a rebuncher, which, used together with the buncher of the third part (working in this case as a second rebuncher), longitudinally matches the beam at the entrance of the cyclotron. The betatronic matching at the object point of the third part is done using a doublet and a triplet.

A sextupole is inserted between the magnets of each pair to compensate for the second order geometrical aberrations.

The first order monochromatic transverse beam envelopes from the object point of the $90^{\circ}$ analysers to the exit of the cyclotron inflector are given in fig. 6 .

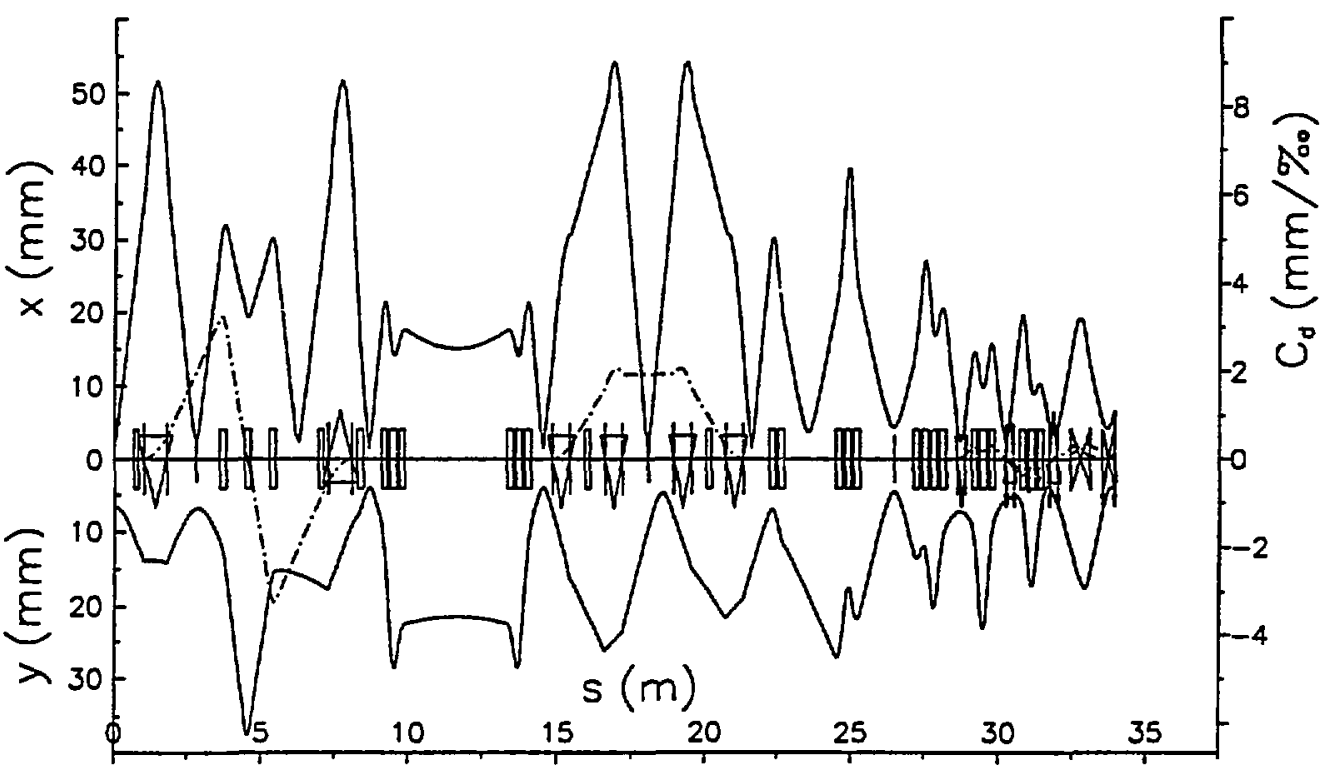

Figure 6 : monochromatic transverse beam envelopes in the low energy beam line when using the LAMS ( $80 \pi \mathrm{mm} . \mathrm{mrad}$ in each transverse plane).

In order to minimize the geometrical aberrations the buncher and the debuncher should be placed in between the two focusing triplets [2] but, to limit the voltages to be applied, they have to be separated by a large enough distance. This was not possible in this first design due to various constraints associated to the general setting up and to the requirements of the downstream optics. This geometry will have to be refined ; nevertheless, the results obtained are quite significant although certainly pessimistic.

In the two cases, either with or without the LAMS, the tuning of the whole line, from the object of the $90^{\circ}$ spectrometers to the injection inside the cyclotron, can be, to first order, independant of the transverse emittance. In this case, the beam envelopes scale as $E_{x}{ }^{1 / 2}$ and $E_{y}{ }^{1 / 2}$. 


\section{4 -SIMULATION OF THE TWO VERSIONS OF THE LOW ENERGY BEAM LINE.}

\section{1- The conditions of the simulation.}

We use the 3D multiparticle code SOSO, developed at GANIL where :

- the initial $\mathrm{c}$-w beam is simulated using test particles uniformly distributed first in a 5D ellipsoid in $x, x^{\prime}, y, y^{\prime}, \Delta w / w$ and then on $2 \pi$ in $\phi$. In the results the beam half width $\Delta x$ on a given axis $x$ is always defined as equal to $2 \sigma_{x}$ of the projection of the $6 \mathrm{D}$ distribution on this axis,

- the equations of motion are integrated step by step in the field of the dipoles which is given on a polar mesh in the median plane, the field components outside of this plane being deduced to first order,

- the beam dynamic in the quadrupoles and the sextupoles is performed using the second order matrixes corresponding to thick elements (as given in TRANSPORT [7] ),

- the buncher, debuncher and rebunchers are considered as thin lenses without any transverse effect, this approximation being well justified for gridded gaps. For the buncher and the rebunchers, the potential across the gap is given by [5] :

$$
V=V_{m}(\sin \phi-0.4 \sin 2 \phi+0.18 \sin 3 \phi-0.06 \sin 4 \phi)
$$

and for the debuncher a simple sine function has been used, this proves to be sufficient since at its location the beam is rather well bunched.

\subsection{The simulated heams.}

The cyclotron energy range ( $2-25 \mathrm{Mev} / \mathrm{A}$ ), using an $\mathrm{RF}$ frequency varying from 9.6 to $14.5 \mathrm{Mhz}$, is covered with the harmonic numbers $\mathrm{h}=2,3,4$ and 5 as shown in fig.2. To each harmonic corresponds a given $\beta \lambda=2 \pi \mathrm{rinj} / \mathrm{h}$.

We choose to simulate 4 cases corresponding to the 4 harmonic numbers i.e to the 4 values of $\beta \lambda$. In each case we assume the maximum magnetic field in the cyclotron and 3 values of the transverse emittances : $20,40,80 \pi \mathrm{mm}$.mrad. The energy dispersion at the ECRIS exit is taken equal to $5 \mathrm{eV}$ per charge : $\Delta \mathrm{w}_{0} / \mathrm{w}_{0}=5 / \mathrm{V}_{0}$.

The data relative to these beams (pointed as * in fig.2) are given in tables 1 and 2. In table 2 we also give the values obtained in the simulation. In the case of the LAMS, a theoretical chopper has been simulated in order to compare with the theory, it limits the beam phase width to the linear part of the buncher and debuncher voltages : $\Delta \phi_{0}=135^{\circ}$.

\begin{tabular}{|c|c|c|c|c|c|}
\hline $\mathrm{h}$ & $\mathrm{Q} / \mathrm{A}$ & $\mathrm{V}_{0}(\mathrm{kV})$ & $\mathrm{f}_{\mathrm{HF}}(\mathrm{MHz})$ & $\beta \lambda(\mathrm{cm})$ & $\Delta \mathrm{w}_{0} / \mathrm{w}_{\mathrm{o}}(\% \mathrm{o})$ \\
\hline 2 & 0.25 & 30 & 12 & 10.02 & 0.167 \\
3 & 0.20 & 24 & 14.4 & 6.68 & 0.208 \\
4 & 0.15 & 18 & 14.4 & 5.01 & 0.278 \\
5 & 0.10 & 12 & 12 & 4.01 & 0.417 \\
\hline
\end{tabular}

Table 1 : the simulated beams. 


\begin{tabular}{|c|c|c|c|c|c|c|c|c|c|c|}
\hline \multirow{2}{*}{\multicolumn{2}{|c|}{$\begin{array}{l}\text { Ex=Ey } h \\
\text { mm.mrad }\end{array}$}} & \multicolumn{4}{|c|}{$90^{\circ}$ spectrometers } & \multicolumn{5}{|c|}{ LAMS } \\
\hline & & $\begin{array}{c}\mathrm{e}_{0} \\
\mathrm{~mm}\end{array}$ & $\begin{array}{c}\Delta \mathrm{w}_{0} / \mathrm{w}_{0} \\
\% \text { oo }\end{array}$ & $\Delta \mathrm{x}_{\mathrm{t}}$ & $\mathrm{m}_{\mathrm{s}}$ & $\begin{array}{l}\mathrm{e}_{0} \\
\mathrm{~mm}\end{array}$ & $\begin{array}{c}\Delta \mathrm{w}_{\mathrm{c}} / \mathrm{w}_{0} \\
\%_{0}\end{array}$ & $\begin{array}{c}\Delta \phi_{\mathcal{c}} \\
\circ\end{array}$ & $\Delta \mathrm{x}_{\mathrm{t}}$ & $\underset{\mathrm{nm}}{\Delta \mathrm{x}_{\mathrm{S}}}$ \\
\hline \multirow[t]{4}{*}{$20 \pi$} & 2 & 1.095 & 0.167 & 1.11 & 1.11 & 0.868 & 1.27 & 58.0 & 1.54 & 1.53 \\
\hline & 3 & & 0.208 & 1.11 & 1.11 & & 1.00 & 57.7 & 1.32 & 1.33 \\
\hline & 4 & & 0.278 & 1.13 & 1.13 & & 0.95 & 59.0 & 1.28 & 1.31 \\
\hline & 5 & & 0.417 & 1.17 & 1.17 & & 1.22 & 62.1 & 1.50 & 1.50 \\
\hline \multirow[t]{4}{*}{$40 \pi$} & 2 & 1.549 & 0.167 & 1.56 & 1.57 & 1.228 & 1.41 & 57.9 & 1.87 & 1.84 \\
\hline & 3 & & 0.208 & 1.56 & 1.57 & & 1.16 & 57.7 & 1.69 & 1.69 \\
\hline & 4 & & 0.278 & 1.57 & 1.58 & & 1.13 & 59.1 & 1.67 & 1.66 \\
\hline & 5 & & 0.417 & 1.60 & 1.61 & & 1.31 & 62.1 & 1.79 & 1.78 \\
\hline \multirow[t]{4}{*}{$80 \pi$} & 2 & 2.191 & 0.167 & 2.20 & 2.22 & 1.736 & 1.75 & 57.9 & 2.46 & 2.32 \\
\hline & 3 & & 0.208 & 2.20 & 2.23 & & 1.56 & 57.8 & 2.33 & 2.16 \\
\hline & 4 & & 0.278 & 2.21 & 2.23 & & 1.60 & 59.2 & 2.36 & 2.20 \\
\hline & 5 & & 0.417 & 2.23 & 2.25 & & 1.69 & 62.3 & 2.42 & 2.29 \\
\hline
\end{tabular}

Table 2: half beam widths at the image points of the $90^{\circ}$ analysers and of the LAMS :

$\mathrm{e}_{0}$ : first order monochromatic values,

$\Delta \mathrm{x}_{\mathrm{t}}$ : theoretical chromatic values,

$\Delta \mathrm{x}_{\mathbf{s}}$ : values as given by the simulation,

$\Delta w_{c} / w_{0}, \Delta \phi_{c}:$ values after the debuncher.

In all the cases, $\Delta x_{S}$ is practically equal to $\Delta x_{t}$ : the effect of the sextupoles is almost perfect. Concerning the beam phase width at the debuncher location and the resulting energy dispersion after its action, they are much larger than the theoretical ones : as already said this is in part due to the rather bad location of the buncher and debuncher.

\subsection{The mass selection.}

The separation in LAMS is illustrated in fig. 7 which shows for 3 values of $\varepsilon$ the central phase shifts, the resulting mean energy shifts just after the debuncher (a pure sinusoidal voltage is used in this case) and the radial separation of the three components in front of the slit.

Table 3 gives the theoretical separation $\left|\delta \varepsilon / \varepsilon_{0}\right|$ obtained either with the first $90^{\circ}$ spectrometers or with the LAMS ; in each case when using LAMS, the radial slit aperture has been chosen to transmit $\cong 75 \%$ of the $\delta \varepsilon=0$ ions. These values have to be compared to the results given by the numerical simulations which are also reported in this table and shown in fig.8. 

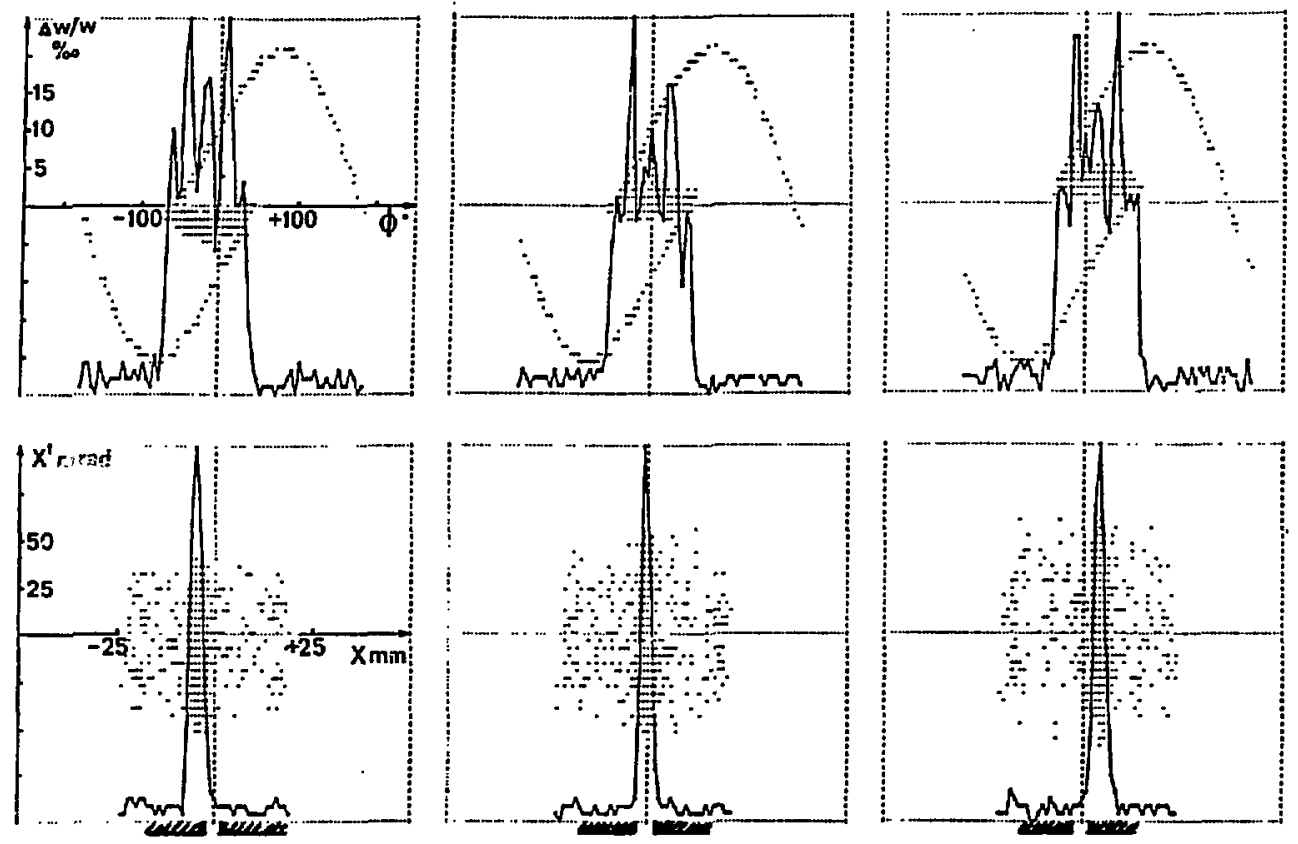

$\mathrm{N}=74$ ions

$N=728$ ions

$\delta \varepsilon / \varepsilon_{0}=0$

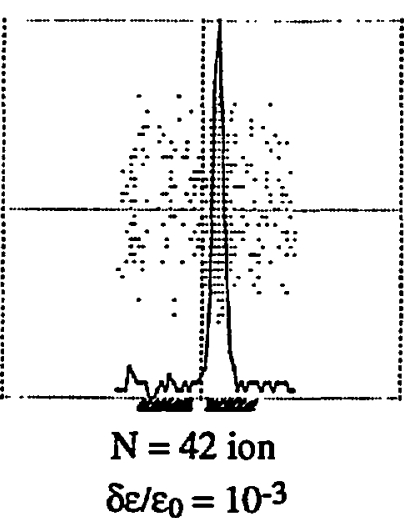

Figure 7 : the separation of $\delta \varepsilon / \varepsilon_{0}=0, \pm 10^{-3}$ ions in LAMS.

Projections of 1000 test particles just after the debuncher $(\Delta w / w-\phi)$ and in front of the radial slit $\left(x^{\prime}-x\right)$. Initial $c-w$ beam $\left(h=3,80 \pi \mathrm{mm} \cdot \mathrm{mrad}, \Delta w_{0} / w_{0}=0.2110^{-3}\right)$.

Number $\mathrm{N}$ of ions crossing a $\pm 2.1 \mathrm{~mm}$ radial slit.

Fig.9 shows the selection obtained for different slit apertures $(h=4,40 \pi$ $\mathrm{mm} . \mathrm{mrad})$. It also illustrates a very simple way for selecting a given $\varepsilon$ : we just move the phase of the debuncher, a much easier, faster and more reproducible operation than the tuning of magnetic dipoles as in classical spectrometers. Shifting the rebuncher phase by $10^{\circ}$ gives an $\cong 4.10^{-4}$ variation in the $\varepsilon$ of the selected ions (theoretically $4.7710^{-4}$ ).

The tails (non linear parts of the buncher voltage) are almost completely removed after the slit, this is due to the large energy dispersion of the ions they contain ; nevertheless, as can be seen in fig. 8 and 9, some ions are not completely eliminated and are responsible for a small background ( $\equiv 2.5 \%$ ), whatever the $\delta \varepsilon / \varepsilon_{0}$ and the slit aperture.

The gain obtained using LAMS is quite obvious, especially in the case of an initial beam having a large emittance and a small energy dispersion. Of course, the beam energy dispersion is increased, but just as it would be when bunching in the same phase width using a corventional bunching system and the transmission given by LAMS is not worse than the capture by a buncher. 


\begin{tabular}{|c|c|c|c|c|c|c|}
\hline \multirow[t]{2}{*}{$\mathrm{h}$} & \multirow{2}{*}{$\begin{array}{c}\Delta w_{0} / w_{0} \\
\%_{0}\end{array}$} & \multirow{2}{*}{$\begin{array}{c}\text { Ex }=E y \\
\text { mm.mrad }\end{array}$} & \multicolumn{2}{|c|}{$\delta \varepsilon / \varepsilon_{0} 90^{\circ}$ Spectro $(\%)$} & \multicolumn{2}{|c|}{$\delta \varepsilon / \varepsilon_{0}$ LAMS $(\% o)$} \\
\hline & & & Theory & Simulation & Theory & Simulation \\
\hline 2 & 0.167 & $20 \pi$ & 2.21 & & 0.51 & \\
\hline 3 & 0.208 & $"$ & 2.23 & always & 0.54 & always \\
\hline 4 & 0.278 & $"$ & 2.26 & $\cong 3$ & 0.61 & $\cong 1$ \\
\hline 5 & 0.417 & $"$ & 2.34 & & 0.77 & \\
\hline 2 & 0.167 & $40 \pi$ & 3.11 & & 0.68 & \\
\hline 3 & 0.208 & $"$ & 3.12 & always & 0.70 & always \\
\hline 4 & 0.278 & $"$ & 3.15 & $\equiv 4$ & 0.76 & $\equiv 1$ \\
\hline 5 & 0.417 & $"$ & 3.21 & & 0.89 & - \\
\hline 2 & 0.167 & $80 \pi$ & 4.37 & & 0.93 & \\
\hline 3 & 0.208 & $"$ & 4.40 & always & 0.95 & always \\
\hline 4 & 0.278 & $"$ & 4.42 & $\equiv 5$ & 0.99 & $\equiv 1.5$ \\
\hline 5 & 0.417 & $"$ & 4.46 & & 1.09 & \\
\hline
\end{tabular}

Table 3 : comparison of the selections as given by the $90^{\circ}$ spectrometers and by the LAMS : $(\mathrm{L} / \beta \lambda)\left(\mathrm{V}_{\mathrm{I}} / N_{0}\right)=(4 / 3)\left(\Delta \phi_{0} / \pi\right)=1$.

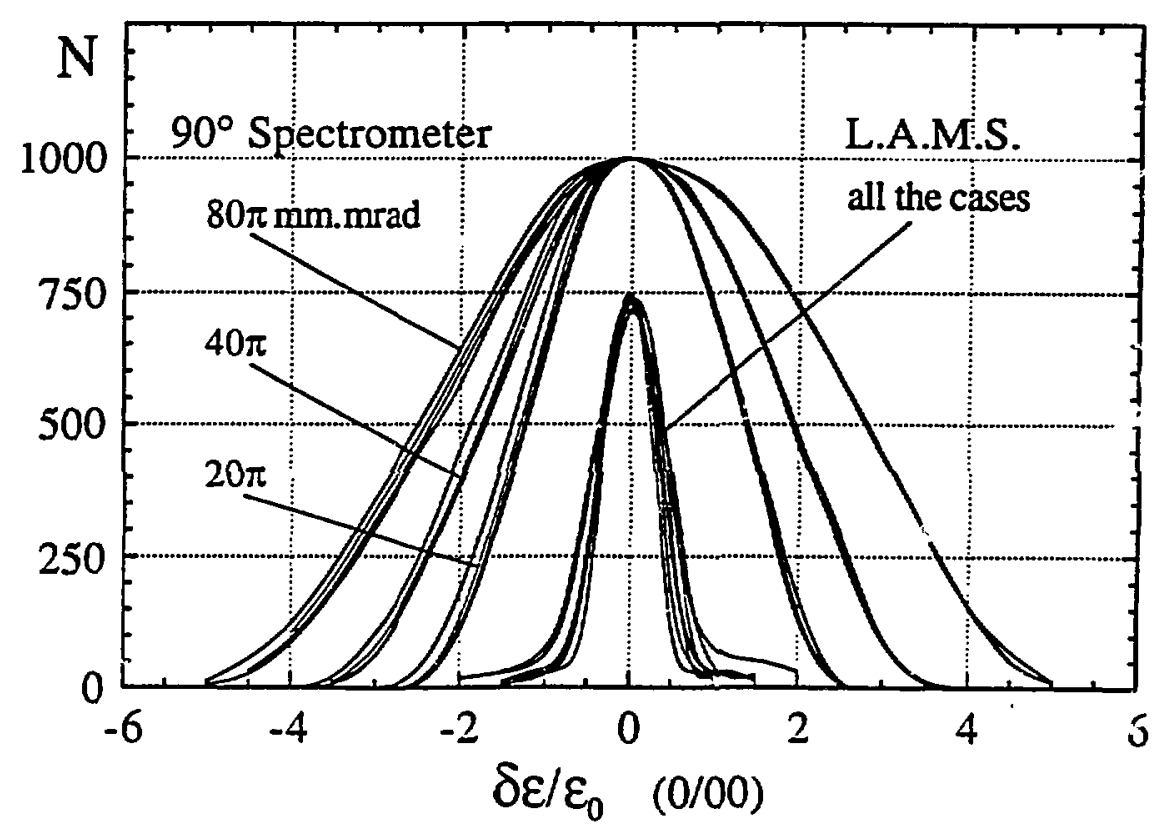

Figure 8 : the separation obtained with the $90^{\circ}$ spectrometers and with LAMS for all the cases listed in table 2 and 3 : number of ions behind the slits opened to transmit in each case respectively $100 \%$ and $\cong 75 \%$ of 1600 initial test particles of $\delta \varepsilon / \varepsilon_{0}=0$. 


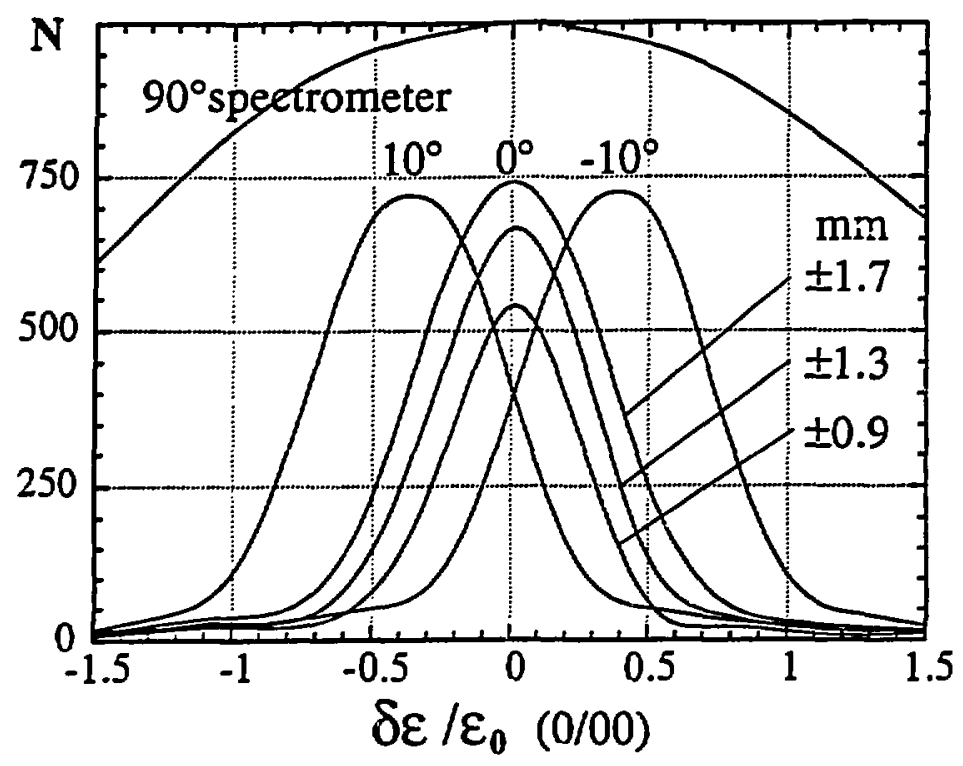

Figure 9 : the selection obtained for various slit apertures and the selected ions for various settings of the debuncher $R F$ phase $(\mathrm{h}=4,40 \pi \mathrm{mm}$.mrad).

\subsection{The particles injected into the cyclotron.}

Fig.10 displays the $\Delta w / w-\Delta \phi$ phase space just behind the cyclotron inflector using or not the LAMS.
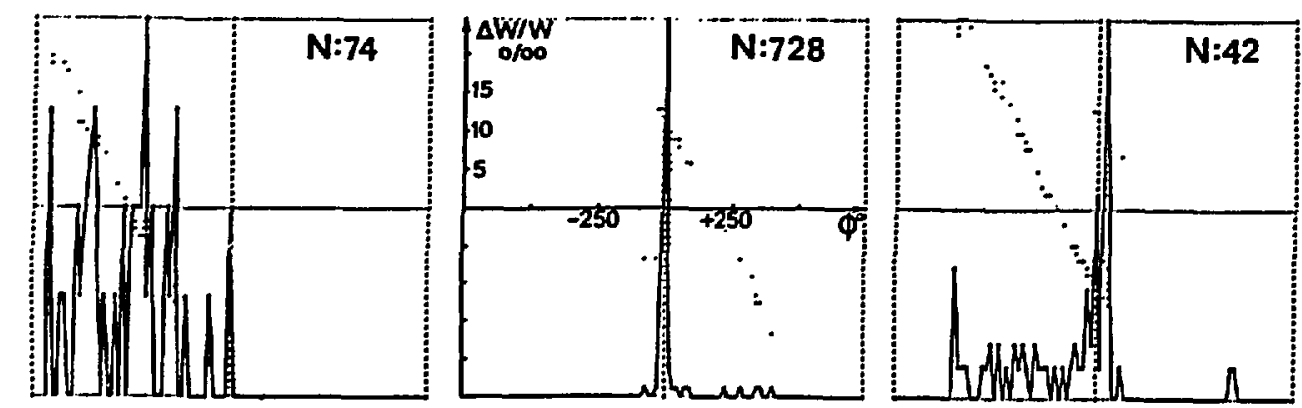

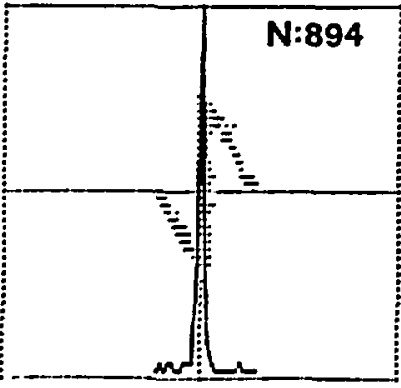

$\delta \varepsilon / \varepsilon_{0}=-10^{-3}$

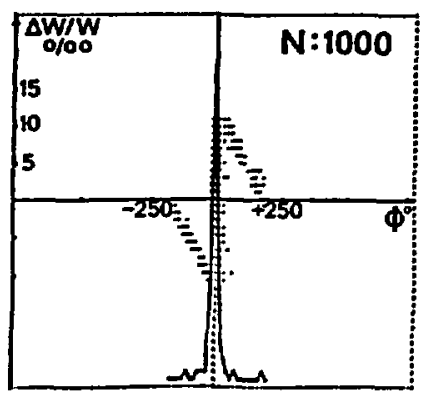

$\delta \varepsilon / \varepsilon_{0}=0$

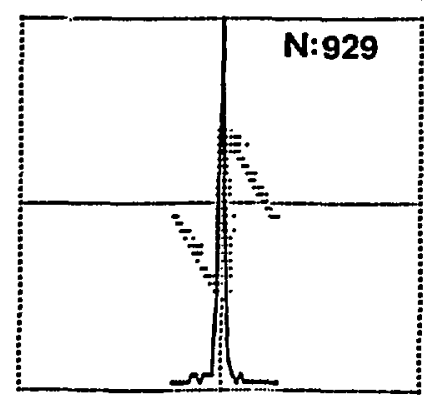

$\delta \varepsilon / \varepsilon_{0}=10^{-3}$

Figure $10: \Delta w / w-\phi$ projections at the inflector exit of the $N$ ions remaining after the selection when using or not the LAMS $(\mathrm{h}=3,80 \pi \mathrm{mm}$.mrad). 
In fig. 11 we have summarized the results obtained for a $80 \pi$ mm.mrad beam accelerated on $\mathrm{h}=3$. This figure shows, versus $\delta \varepsilon / \varepsilon_{0}$, the ions selected by the image slits of the spectrometers and the number of particles contained, behind the inflector, in a $\pm 6^{\circ}$ phase width around the central phase of the $\delta \varepsilon=0$ ions.

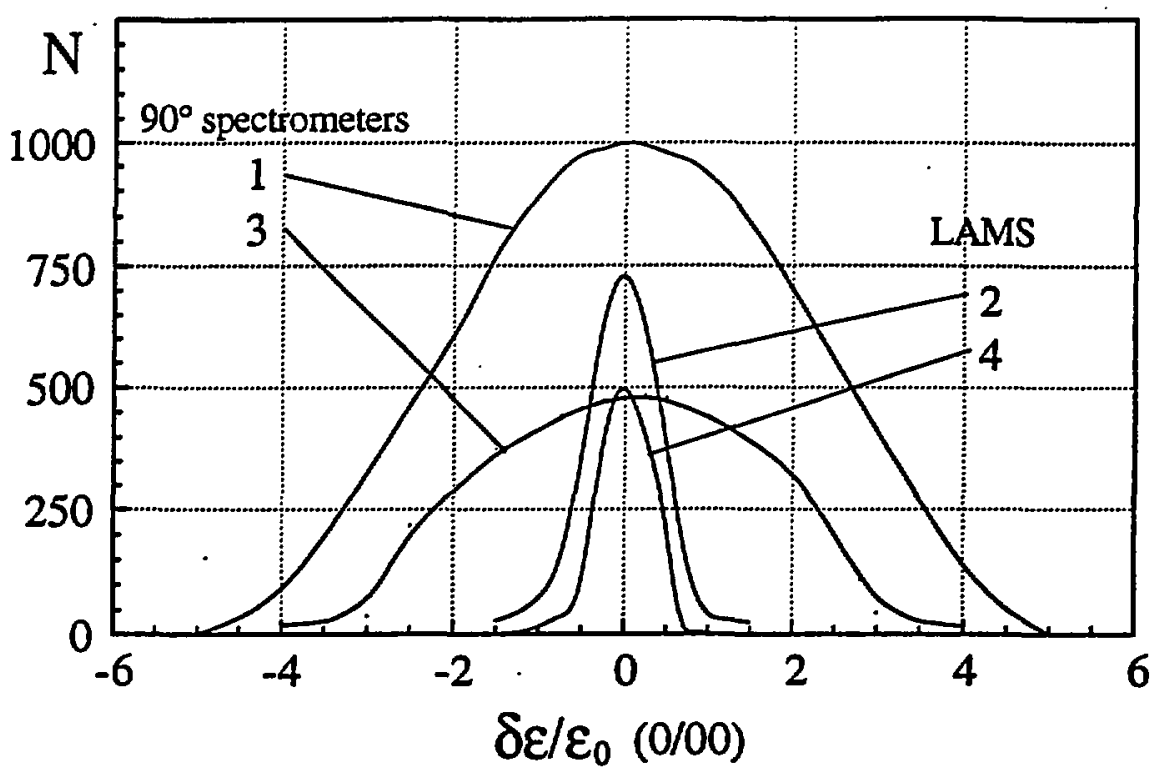

Figure 11 : the selection using (2) or not (1) the LAMS and the number of ions in $a \pm 6^{\circ}$ phase width behind the inflector using (4) or not (3) the LAMS $(\mathrm{h}=3,80 \pi \mathrm{mm} \cdot \mathrm{mrad})$.

In the two cases, all the ions remaining after the slit selection are injected into the cyclotron. The RF phase of the accelerator being locked on the central phase of the $\delta \varepsilon=0$ ions we want to accelerate, particles having a phase 'far enough' from the central one will be deccelerated or, if not, lost on the very first turns.

After the inflector, the phase shifts between the central phases of each kind of ions are proportionnal to the geometrical distance from the first buncher they have crossed to the inflector exit. On the other hand, the shortest the distance between the last rebuncher and the inflector exit, the smallest the phase width the ions are bunched in. The differences between the two cases are related to these remarks.

Using or not LAMS, the last rebuncher or the buncher respectively, located as near as possible of the cyclotron, will give the same bunching efficiency for $\delta \varepsilon=0$ ions. Without LAMS, the buncher acts on a cw beam, that means almost in the same way on all the ions whatever their $\varepsilon$; being located rather close to the inflector exit, all the ions not eliminated by the $90^{\circ}$ spectrometer will enter the cyclotron with nearly the same central phases ; they will be accelerated during a number of turns depending on their $\delta \varepsilon$ value. Using LAMS, we first have a much better selection at the image slit but moreover, at the entrance of the last rebuncher, the various kinds of ions are already bunched around 
different central phases : the rebuncher acts differently on each species and this will greatly contribute to the beam purification.

\section{5 - CONCLUSIONS.}

In the simplest version we have presented, the LAMS is characterized by its large acceptance ( up to $\cong 100 \pi \mathrm{mm}$.mrad ) and its simplicity. It is well suited to the beams of low energy spread which are given by the ECR ion sources and its insertion in our RIB facility should allow :

- a much more precise and easy identification of the various kinds of ions which are extracted from the ECRIS,

- a better selection of the required isotope and the optimization of its production,

- a limitation of the number of species injected into the last part of the low energy beam line and into the accelerator thus reducing all the problems related to the tuning and those coming from the distributed losses of unwanted radioactive particles,

- a monitoring by using a spare kind of ions representative of the production of the isotope we want to accelerate.

Radioactive ions being very difficult and expensive to produce, it would certainly be of a great interest to provide for experiments a spare kind of ions at the ECRIS extraction energy ; this could easily be done at the slit location.

For the time being, our proposal for inserting LAMS is not funded ; if it was, this study would have to be refined in view of reducing the non linear effects as much as possible, in particular we would place the buncher and the debuncher in between the focusing elements of the drift. It would also be necessary to pay a special attention at the tolerances on the magnetic field of the dipoles and to take into account the real effects in the bunching elements.

\section{6- REFERENCES}

[1] 'SPIRAL : The R.I.B. Facility under construction at GANIL', M. Lieuvin and the SPIRAL Group, presented at the 4th EPAC, London 1994.

[2] 'Principle of a new kind of large acceptance mass separator', A.Chabert, Ch.Ricaud, B.Bru, GANIL A 94 01, to be published in NIM.

[3] 'Six dimensional beam matching for axial injection into a cyclotron', R. Beck, S. Chel, B. Bru and Ch. Ricaud, Proc. of the 12th Int. Conf. on Cyclotrons and their Applications, Berlin, 1989, 432 - 434.

[4] 'Design of a beam buncher using a half-cosine driving function', R.C.Rogers, Proc. of 13th Int. Conf. on Cyclotrons and their Applications, Vancouver 1992, 629-633. 
[5] 'Beam buncher for heavy ions', F.J.Lynch, R.N.Lewis, L.M.Bollinger, W.Henning and O.D.Despe, Nucl. Instr. and Meth. 159 (1979) 245-263.

[6] 'GALOPR, a beam transport program with space charge and bunching', B. Bru, Proc. of the 3d CPO Conference, Toulouse 1990, p 27 - 34.

[7] 'A first and second order matrix theory for the design of beam transport systems and charged particle spectrometers', K.L.Brown-SLAC-75 (1967). 